Literature Reviews

\title{
Clinical Uses and Molecular Aspects of Ozone Therapy: A Review
}

\author{
Ana Paula Pivotto, Fernanda Weyand Banhuk, Izabela Virginia Staffen, \\ Maiara Aline Daga, Thaís Soprani Ayala and Rafael Andrade Menolli \\ Laboratory of Applied Immunology, Center of Medical and Pharmaceutical Sciences, \\ UNIOESTE-Western Parana State University, Cascavel/PR, Brazil
}

\author{
Article history \\ Received: 07-01-2020 \\ Revised: 12-02-2020 \\ Accepted: 24-02-2020 \\ Corresponding Author: \\ Rafael Andrade Menolli \\ Laboratory of Applied \\ Immunology, Center of \\ Medical and Pharmaceutical \\ Sciences, UNIOESTE-Western \\ Parana State University, \\ Cascavel/PR, Brazil \\ Email: rafael.menolli@unioeste.br
}

\begin{abstract}
Ozone was discovered over a hundred years ago and since then, it has been widely used in many areas, with the primary use as a disinfectant in various ways, but with applications in diverse conditions. More recently, the use of ozone has extended to other fronts, using it to treat different pathologies. Extensive studies have shown its effects, as well as the safety of its application in various modalities of use. Other studies showed its toxicity, which depends on the doses of use. Emerging evidence revealed that ozone also plays an important role in the wound healing and modulation of immune cells, describing the molecular pathways responsible for these actions and describing therapeutic actions in the treatment of wounds, pain, postoperative and infectious diseases. Ozone use has already been documented in different doses, forms of use and routes of application, depending on the clinical situation and an adaptation is necessary for a better result. Thus, this review summarizes the main clinical uses of ozone, presenting the molecular pathways responsible for its actions, as well as discussing the main routes of use, doses and, vehicles used in the clinic.
\end{abstract}

Keywords: Ozone, Mechanism of Action, Immunomodulation, Antioxidant, Wound Healing

\section{Introduction}

Ozone $\left(\mathrm{O}_{3}\right)$ is an allotrope of oxygen, which at room temperature, is an explosive gas that absorbs UV radiation in the range $220-290 \mathrm{~nm}$ (Eriksson et al., 2007). Ozone is a gas with a pungent and characteristic smell. It is formed through electrical discharges on the oxygen molecule, which breaks down, releasing atoms that bind to the other two molecules, generating $\mathrm{O}_{3}$. At high concentrations in air, it turns blue, while at low concentrations, it is a colorless gas, lighter than the air (Wysok et al., 2006). Ozone loses only to fluorine in its oxidizing power and oxidizes most inorganic compounds to their final oxidative state, converting ferrous, manganous and chrome ions to their respective higher oxidation states (Loegager et al., 1992). Because it is exceptionally oxidizing and unstable, ozone quickly returns to its molecular oxygen form, but for medical use, it needs to be synthesized through specific generators. Most medical generators on the market use the corona effect to produce the oxygen-ozone gas mixture. Some studies demonstrate the generation of ozone in the antigen-antibody complex in the human body, which proves that the production of this molecule happens physiologically via the immune system too (Nathan, 2002).

Ozone is an unstable molecule, so it is usually mixed with oxygen in ozone therapy (Biçer et al., 2016). This product has powerful oxidizing properties, forming oxygen-free radicals that lead to the destruction of microorganisms and improves stimulation of the process of healing. As a result, the immune system is activated to fight pathogens such as bacteria, fungi and virus infections (Stübinger et al., 2006). Ozone therapy is effective in managing many skin conditions, mainly wound healing, primarily because of its ability to promote inflammation in the body such as the secretion of immunomodulatory products and, reduction of the oxidative damage. Ozone therapy has been widely studied in infected wounds, gangrene, burns and, circulatory disorders, showing to be highly effective in the outcome of these conditions (Gulmen et al., 2013). Its characteristics as an activator of the antioxidant system make this molecule capable of stimulating tissue healing and an anti-inflammatory environment at low 
doses, but when used in high concentrations, it can lead to an exacerbation of inflammation (Bocci et al., 2011).

Thus, this article aims to review the main aspects of ozone therapy regarding its clinical uses, routes of administration, toxicity, as well as the molecular mechanisms, highlighting the benefits and also the main challenges and difficulties of its use.

\section{Uses of Ozone}

Ozone was first suggested as a potable water disinfectant in the nineteenth century because of its powerful ability to inactivate microorganisms (Ohmuller, 1892) and it has been later confirmed by other researchers that this substance, when used for limited periods, can effectively disinfect the water supply (Broadwater et al., 1973). It has been considered an effective alternative antimicrobial agent to chlorine, even with adverse toxicological problems arising from its use in high concentrations (Morris, 1971).

Due to its ability to oxidize substances and destroy microorganisms, ozone has been widely used to sterilize air, clean water and remove odors. In addition, its application is intended for medical use. Ozone therapy has been used and studied extensively for many decades. It has been used to treat infections, wounds and various diseases (Elvis and Ekta, 2011).

It was considered as an autohemotherapeutic agent in the treatment of HIV-infected patients in the 1980s (Garber et al., 1991), however, subsequent studies have proven that ozone therapy neither improves nor worsens the dynamics of HIV-1 replication in vivo (Bocci et al., 1998).

Although ozone has been applied to treat over 100 diverse diseases with varying results, the supporting evidence for most medical ozone applications is limited. In 1976, inhalation of ozone gas was able to induce lung inflammation and pulmonary edema, and this led the US Food and Drug Administration (FDA) to declare concern about its toxicity, which was reiterated in 2006 (USFDA, 2019). The FDA also emphasized that effective therapeutic ozone concentration should be as far as possible from tolerable doses by humans and other animals (Wang, 2018).

Ozone has wide medical applications due to its effective antimicrobial function (Ozturk et al., 2017), antioxidant defense and the anticipation in wound healing (Delgado-Roche et al., 2017; Elvis and Ekta, 2011; Mirowsky et al., 2016) and in the control of bleeding (Gupta and Mansi, 2012). The effects on immunoregulation and antioxidant defenses, as well as the effectiveness of ozone therapies, have been gradually recognized, although the mechanisms involved in various conditions are still unclear. Due to its role as an activator of antioxidant defenses, ozone is widely used for cosmetic-related services (Borrelli et al., 2012) and general health services (Di Paolo et al., 2005).
In another direction, depending on the exposure time and dose, ozone may promote harmful effects to the individual, knowing that inhalation of ozone gas, as well as its intake, is harmful to health (Bocci et al., 2012), as well as attaining motor and cognitive activity, leading to brain dysfunction and memory loss, seen in experiments with rats exposed to ozone (Rivas-Arancibia et al., 1998).

\section{Route of Administration}

Many clinical and laboratory in vitro and in vivo animal studies have shown the effectiveness of ozone therapy used in several pathways (Gautam et al., 2011; Izadi et al., 2019; Martínez-Sánchez et al., 2005). Generally, ozone therapy was conducted by introducing the mixing of ozone with gases or liquids into the body through various ways, including intravaginal, intrarectal, intramuscular, subcutaneous or intravenous routes (Wang, 2018). Ozone has hemodynamics and can also be introduced into the body through autohemotherapy (Garber et al., 1991).

The topical route is the main route used for wounds and ulcers, such as those occurring in the diabetic foot (Izadi et al., 2019; Martínez-Sánchez et al., 2005) and also in cases of burns (Campanati et al., 2013). Also, the use of topical ozone therapy was widely used in dental procedures (Sivalingam et al., 2017). The primary vehicles for topical uses are water, saline, or oil and the ozonation of these vehicles can occur before or during the procedure, the area to be treated can be submerged in the ozonated vehicle or spread/applied directly over the area (Campanati et al., 2013; Izadi et al., 2019).

Parenteral ozone therapy encompasses systemic and local injection and is widely used mainly in the treatment of low back pain, arthritis, coronary artery disease, herniated disc, osteoarthritis, hepatitis $\mathrm{C}$, muscle pain, among other conditions (Babaei-Ghazani et al., 2018; Celakil et al., 2017; Gautam et al., 2011; Martínez-Sánchez et al., 2005; Zaky et al., 2011). Beyond the practices of systemic ozone therapy are rectal insufflation, as in the case of coronary artery disease and also in the treatment of hepatitis C (Martínez-Sánchez et al., 2012; Zaky et al., 2011) and intravenously, performing autohemotherapy to treat the symptoms of hepatitis C too (Zaky et al., 2011) and also in cases of diabetic foot (Izadi et al., 2019). Intramuscular infiltration for acute spinal pain (Paoloni et al., 2009), intradiscal for lumbar disc herniation (Gautam et al., 2011) and intrapatellar injection for osteoarthritis treatment in the knee (BabaeiGhazani et al., 2018) could be classified as local injections but not systemic or topical applications.

\section{Vehicles for the Use of Ozone Therapy}

Ozone therapy is available through different types of vehicles. It is commonly used in the form of gas, under 
topical treatment, where the skin is covered with a plastic bag filled with ozone gas-oxygen mixture (Wang, 2018). It also can be used in the form of ozonated saline, which consists of prior saturation of physiological saline with an oxygen-ozone mixture (Clavo et al., 2013).

An ozone generator is used when the aqueous ozone is needed, which mixes ozone with water. This format is highly valid for many skin-related disorders and is widely used to treat infectious diseases. However, the disadvantage of ozone hydrotherapy is the short shelf life and its instability, which makes this treatment an option available only in equipped clinics (Wang, 2018). Its short half-life is approximately 20 minutes in water at $20^{\circ} \mathrm{C}$, it is partially soluble in water and, like most gases, increases its solubility as the temperature decreases (Kim et al., 1999; Wysok et al., 2006). This solubility of ozone in aqueous media will depend on the content of organic matter in the medium, as the lower the concentration of organic matter, the longer the half-life of ozone in water. The decomposition of ozone in the liquid phase is characterized by a rapid decrease in initial concentration, with a later phase in which ozone concentration decreases according to first-order kinetics, with hydroxyl radicals $(\mathrm{OH})$ being the main products of this decomposition (Almeida et al., 2004; Bocci, 2004).

Ozone can react with organic compounds in aqueous solution by a direct ozone reaction or by an indirect reaction of $\mathrm{OH}$ radicals, formed from the decomposition of ozone. This indirect reaction can promote an attack on organic compounds approximately 100 times faster than some oxidizing agents such as $\mathrm{H}_{2} \mathrm{O}_{2}$ and ozone itself. Predominantly, the disinfection processes occur via molecular ozone, whereas the oxidation processes can occur through both direct and indirect processes (Almeida et al., 2004; da Silva et al., 2011).

Ozonated water is applied to wounds and ulcers, at different concentrations depending on the outcome expected (disinfect or regenerate) and the type of tissue to which it will be applied (Clavo et al., 2013). Ozonated water is indicated for pain relief, disinfection and antiinflammatory effects in acute and chronic lesions, with or without infection (Wang, 2018).

Another option is the ozonated oil, obtained by bubbling ozone into cold-pressed oils such as olive oil, sunflower oil and many other unsaturated fatty acids (Menéndez et al., 2011). It has the advantage of the shelf life of 2 years if stored in refrigeration and the topical application of this formulation can be performed at home, which decreases the visits of the patients to the medical institutions. Ozone oil is used in many conditions, including wounds, anaerobic, viral and fungal infections, as well as ulcers, anal fissures, and vulvovaginitis, without any visible side effects (Bocci, 2004).

The vehicle by which ozone is applied can also play a role in the mechanism of ozone therapy. Ozonated oils and water act as stimulators, with both forms improving blood circulation, oxygen delivery, and regularized antioxidant enzymes to activate the immune system and promote the release of growth factors. These phenomenons happen because ozone reacts with the polyunsaturated fatty acids present in the stratum corneum to generate reactive oxygen species and lipo-oligopeptides, substances that can be partially absorbed by skin, capillary and lymphatic antioxidants (Sega et al., 2010).

Direct ozonation of vegetable oils with unsaturated fatty acids leads to the formation of the 1,2,4-trioxolane portion (Sega et al., 2010), which represents an active form of ozone in these materials. The trioxolane ring in the ozonated vegetable oil rapidly produces compounds that accelerate the healing process in wet wounds or ulcers and are responsible for antimicrobial and antimycotic treatments (Menéndez et al., 2011). In addition, the oil itself can act as a moisturizer and protector, especially for patients with the compromised skin barrier.

Studies using vegetable oils in their pure form have found that oils obtained from plants popularly used as healers have demonstrated their healing effect. In a study by Oliveira et al. (2013), it was found that pumpkin oil increased tensile strength and increased collagen synthesis, possibly as a result of the action of fatty acids (Oliveira et al., 2013). The sunflower seed oil has positively contributed to wound healing (Coelho et al., 2012; Rodrigues et al., 2004). Oleic and linoleic acids, the main components of sunflower oil, also present in extra virgin coconut oil, demonstrated that when topically applied, promoted rapid and satisfactory healing in mice (Cardoso et al. 2004).

\section{Ozone Clinical Uses}

Ozone therapy has been used to treat different conditions as summarized in Table 1, seen in different Randomized Clinical Trial (RCT) and reviewed by many authors. The conditions already studied includes: Tissue healing from inflammatory processes showed by a systematic review that included only RCT for chronic wounds (Fitzpatrick et al., 2018) and revised by Travagli et al. (2010) and Valacchi et al. (2005); for back pain revised by Braidy et al., 2018 and also showed by Andrade et al. (2019) in a RCT; osteoarthritis showed in a systematic reviews (Anzolin and Bertol, 2018; Noori-Zadeh et al., 2019) and seeing in an RCT (Babaei-Ghazani et al., 2018; Lopes de Jesus et al., 2017) and in a clinical trial phase 2 (Calunga et al., 2012); diabetic foot ulcers as showed by a RCT (Zhang et al., 2014) and revised by others (Braidy et al., 2018); dental problems such as caries and periodontitis summarized in a review (Almaz and Sönmez, 2015); in the treatment of systemic sclerosis, as seeing in a RCT (Hassanien et al., 2018); multiple sclerosis, seeing in a clinical trial phase 1 (Delgado-Roche et al., 2017; Nowicka, 2017); among other conditions showed in clinical trials (Vanni et al., 2016). 
Table 1: Ozone therapy results in different clinical conditions

\section{Condition}

Wound healing

Gingival graft surgery

Cardiovascular diseases

Chronic inflammatory diseases

Balanitis xerotica obliterans

Atopic dermatitis

Osteoarthritis

Multiple and systemic sclerosis

Asthma

\section{Outcome}

Increase in vessel numbers, collagen deposition and myofibroblasts numbers; Stimulation of the growth factors and peroxide releases; Improvement in oxygenation of the affected area. Improvement in the healing process; Diminishment of the pain intensity.

Increase in oxygenation in ischemic sites

Improvement of the standard treatment; Reduction in pain sensation.

Anti-inflammatory effect; Reduction in the levels of the gene transcription of cytokines; Reduction in the TG2 enzyme.

Reduction in the exudation, improvement of the erosion process.

Positive effect on the intra-articular redox balance; Pain reduction.

Decrease inflammatory process and cellular oxidative stress; Improved joint mobility;

Reduction in the thickness of the skin.

Reduction in immunoglobulin (Ig)E and HLA-DR levels; Improvement of lung function reduction of the symptoms related.

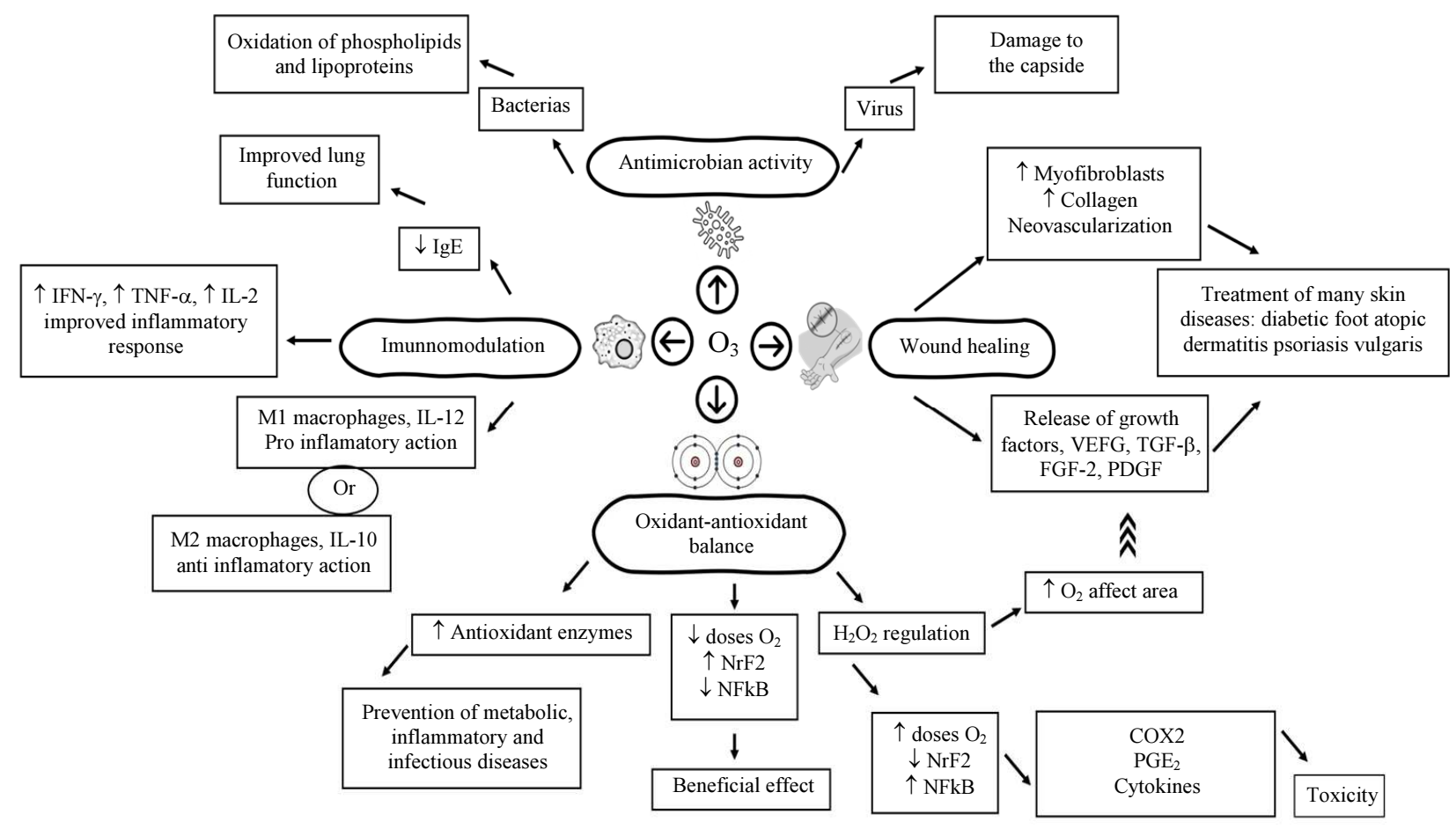

Fig. 1: The main mechanism behind ozone treatment

Ozone therapy is considered an alternative therapy and has several positive effects, but depending on the dose, it may result in harmful effects for the individual (Travagli et al., 2010). The primary reported effects in humans are based on three main functions: Antimicrobial (Ozturk et al., 2017), in the antioxidant/oxidant balance (Calunga et al., 2012) and immunomodulatory effects (Zeng and Lu, 2018) (Fig. 1). It is as a topical treatment that ozone therapy has been most widely used (Solovăstru et al., 2015; Travagli et al., 2010; Zeng and $\mathrm{Lu}, 2018$ ), as ozone gas is highly toxic to the airways, whereas and in skin, a transient exposure at low and controlled doses (range from 10 to $50 \mu \mathrm{g} / \mathrm{mL}$ ), may be beneficial (Valacchi et al., 2005). In the different scenarios of different RCT, the use of ozone acts mainly to anticipate the healing process, such as reducing the size of the diabetic foot injury in individuals with different wound sizes (Zhang et al., 2014), as well as improving the treatment and complete closure of these wounds in association with usual treatment (Wainstein et al., 2011). It also showed this characteristic in the effective healing of the treatment of digital ulcers of patients with systemic sclerosis, improving from $44 \%$ to $96 \%$ the effectiveness of the treatment when combining calcium channel blocker with topical aqueous ozone (Hassanien et al., 2018).

Another RCT showed that the application of ozone on the gingival graft surgery site improved the patient's 
quality of life, as well as improved healing along with lower pain intensity (Taşdemir et al., 2016). Ozone, due to its antioxidant effect (Vanni et al., 2016), has had positive effects in the treatment of cardiovascular diseases, improving the patient's prognosis, such as increasing oxygenation in ischemic sites, as has been demonstrated by a RCT to be beneficial in coronary artery disease and heart failure (Martínez-Sánchez et al., 2012) and showed in a clinical trial to treat peripheral arterial disease (Coppola et al., 2002), besides, reviewed by Mauro et al. (2019), ozone also showed to be beneficial in the prevention of ischemic damage in an in vitro experiment of ischemia (Frosini et al., 2012) and in a myocardial infarction animal model (Di Filippo et al., 2010).

In chronic inflammatory diseases, the use of ozone therapy as an adjuvant has been shown to improve the reference treatment of back pain in patients studied under an RCT (Andrade et al., 2019; reviewed by Bocci et al., 2015). Another clinical practice is in the treatment of disc herniation, which consists in injecting a gaseous oxygen-ozone mixture into the intrasomatic space, site of the disc herniation, or paravertebral muscle, showed in an RCT to be beneficial to the patients, decreasing the pain mainly. This practice was successful in treating this condition (Paoloni et al., 2009). In 2011, a case report of oral osteonecrosis was treated with an ozonated oil suspension, achieving therapeutic success effectively and safely (Ripamonti et al., 2011).

Ozone therapy has also been used in diseases such as Balanitis Xerotica Obliterans (BXO). An RCT with male children showed that ozonized olive oil produced a powerful anti-inflammatory effect demonstrated by the reduction in the levels of gene transcription of cytokines examined in the treated tissues. In addition, it was discovered that ozone treatment was able to reduce the expression of the levels of the Transglutaminase 2 (TG2) enzyme, supporting its anti-inflammatory role in the foreskin affected by BXO (Currò et al., 2018).

Recent data shows the use of ozone also in autoimmune diseases, such as atopic dermatitis and psoriasis vulgaris. An RCT divided sixty children into two groups, the first received ozonated water 3 to 5 times a week and ozonized oil twice a day, the second group received only base oil and a moisturizer for 2 weeks. After 3-5 days of topical ozone therapy, the exudation of the skin was reduced, and the erosion healed. The effective rates were $80.0 \%$ and $20.0 \%$ in the treatment and control group for 1 week and $89.6 \%$ and $30.7 \%$ for 2 weeks, respectively, with a significant difference between the 2 groups $(\mathrm{P}<0.001)$, these data show the effectiveness of topical ozone therapy in this condition (Qin et al., 2018). A clinical trial phase 1 shows the efficiency of ozone in the treatment of psoriasis vulgaris, in which 39 patients whit this condition received medical ozone therapy during the nursing intervention. The cure rate was $38.5 \%$ and the total efficiency was $84.7 \%$, showing that this substance can be also a good alternative in the treatment of this disease (Le, 2012).

An RCT to study the effect of ozone as a therapy to treat osteoarthritis also showed to be a successful approach to treat and relieve the pain of the patients under this condition (Babaei-Ghazani et al., 2018; Lopes de Jesus et al., 2017). Also, a clinical trial showed that a combination of the usual treatment to knee osteoarthritis with ozone therapy had a positive effect on the intra-articular redox balance, reducing the pain of these patients, increasing their quality of life (Calunga et al., 2012).

Ozone was also beneficial to treat multiple sclerosis and systemic sclerosis (Delgado-Roche et al., 2017; Nowicka, 2017). In a clinical trial phase 1, Delgado-Roche et al., 2017, showed that ozone therapy decreases inflammatory process and cellular oxidative stress, promoting a possible neuroprotective effect, where ozone therapy could be used together with the usual treatment, diminishing its toxicity (Delgado-Roche et al., 2017). Also, in a systemic sclerosis clinical trial, Nowicka (2017) presented results where ozone, alone or together with the usual treatment, improved the clinical parameters and skin temperature of these patients, increasing the movability of the joints and decreasing the thickness of the skin (Nowicka, 2017).

\section{Safety Dose and Clinical Protocol}

The safe dose of ozone is variable depending on the location of therapy and the goal of the treatment (Smith et al., 2017). Currently, it has been proposed that ranging from 10 up to $80 \mu \mathrm{g} / \mathrm{mL}$ of ozone, is beneficial leading to the positive effects and no toxicity, depending on the tissue, shown in in vitro and in vivo in animal models (El-Sawalhi et al., 2013; Frosini et al., 2012; Orakdogen et al., 2016; Thiele et al., 1997; Sweet et al., 1980; Smith et al., 2017). In humans studies, the safe dose range varies between 15 and $50 \mu \mathrm{g} / \mathrm{mL}$ and it will depend on the type and goal of treatment as well as the local of application.

Several clinical protocols have been used to treat the different pathologies already mentioned here. Depending on the extent of the disease and the goal of the treatment, the protocol may vary from one week up to months. In the case of diabetic foot ulcers, many studies use ozone treatment in conjunction with conventional treatment (Liu et al., 2015). Clinical studies perform submersion treatment of the lower limb in a bag of water and constant ozonation for $30 \mathrm{~min}$, at an approximate concentration of $50 \mu \mathrm{g} / \mathrm{mL}$ (Izadi et al., 2019; Martínez-Sánchez et al., 2005; Zhang et al., 2014). Different studies have different endpoints, some of them, in the case of the diabetic foot or 
open wounds, treat until complete wound closure or until the reepithelialization process is identified (Izadi et al., 2019), others establish a limit of days, such as 20 consecutive days or so (Zhang et al., 2014). Besides, the use of ozone oil is widely used in topical therapy and the preparation is spread over the wound and left to act for 30 minutes, at an average concentration of $40 \mu \mathrm{g} / \mathrm{ml}$ (Fitzpatrick et al., 2018).

To treat disc herniated low back pain and acute back pain, patients received for five consecutive weeks, three intramuscular injections of an oxygen and ozone mixture at an average concentration of $20 \mu \mathrm{g} / \mathrm{mL}$ (Paoloni et al., 2009). Protocols that perform rectal insufflation, generally indicated for the treatment of low back pain, osteoarthritis, arthritis, multiple sclerosis, among others, use an ozone concentration of approximately $20 \mu \mathrm{g} / \mathrm{mL}$ during approximately 2-3 times per week for 3 months (Anzolin and Bertol, 2018; Delgado-Roche et al., 2017) and also 20 applications for 4 weeks in the case of rheumatoid arthritis (León Fernández et al., 2016). In the case of coronary artery disease, a concentration of 40 $\mu \mathrm{g} / \mathrm{mL}$ once a day for 20 days was effective in decreasing oxidative biomarkers generally present in this condition (Martínez-Sánchez et al., 2012). The autohemotherapy protocols use the patient's collected blood, which is then ozonated at a dose of $20 \mu \mathrm{g} / \mathrm{mL}$ and reinserted intramuscularly or intravenously, as in the treatment of complications of hepatitis C (Zaky et al., 2011) and individuals with peripheral arterial disease (Di Paolo et al., 2005).

In local injection, such as intradiscal injection, used mainly for back pain such as disc herniation, an average of $50 \mu \mathrm{g} / \mathrm{mL}$ of ozone is usually injected into an ozone/oxygen mixture (Niu et al., 2018). Also, there are intrapatellar ozone injection protocols at an average concentration of $15 \mu \mathrm{g} / \mathrm{mL}$ that is effective in treating osteoarthritis (Babaei-Ghazani et al., 2018).

\section{Molecular Mechanisms of Action}

The mechanism of action of ozone therapy lays mainly in three aspects: Immunomodulation (Bocci, 2006; 2007), antimicrobial activity (Polydorou et al., 2012; Thanomsub et al., 2002) and the capacity to interplay with the oxidant/antioxidant balance (Soares et al., 2019). Altogether, another main aspect of the mechanism of the ozone treatment is the wound healing property and the main mechanism behind this is the capacity to interact with the skin epithelization system (Borges et al., 2017; Eroglu et al., 2018), as summarized in Fig. 1.

Ozone therapy, especially in its topical forms, has been used clinically as an adjuvant in wound healing (Borges et al., 2017). Ozone promotes an increase in the epithelialization process increasing matrix deposition and cell proliferation. Ozone-treated wounds also demonstrate a granulation tissue with fewer inflammatory cells and a higher number of myofibroblast-compatible spindle cells. As well as increased vessel numbers and collagen deposition (Soares et al., 2019). Several endogenous growth factors, such as vascular Endothelial Growth Factor (VEGF), Transforming Growth Factor-beta (TGF- $\beta$ ), Fibroblast Growth Factor (FGF) and PlateletDerived Growth Factor (PDGF), play an essential role in early wound healing (Eroglu et al., 2018), so it becomes crucial for the healing process the stimulation of the expression of these growth factors.

Another mechanism by which ozone leads to wound healing involves its interaction with wound exudates that lead to the breakdown of ozone into peroxides and stimulates tissue repair, improving oxygenation in the affected area. Oxygen-responsive species stimulate platelet aggregation and lead to the release of growth factors, that as mentioned above, play a key role in wound healing (Wang, 2018). According to recent researches, ozone increases endogenous growth factors (Eroglu et al., 2018) such as the expression of VEGF, TGF- $\beta$ and PDGF, all involved in the cicatrization process (Zhang et al., 2014). Fibroblast Growth Factor 2 (FGF2), also known as basic FGF, is an essential protein that activates myofibroblastic angiogenesis-associated pathways and proliferation (Svystonyuk et al., 2015). FGF2 expression was associated with fibroblast activation, resulting in higher tissue collagen production. Soares et al. (2019), who used ozone to treat skin wounds in rats, observed that FGF2 was overexpressed in macrophages and myofibroblasts, which may indicate an improvement in wound healing events. This finding corroborates with the hypothesis that these cells, under topical ozone treatment, secrete growth factors and cytokines that are essential to the healing process. Besides, ozone has been reported to induce an increase in the number of macrophages associated with a higher number of myofibroblasts, as well as proliferative neovascularization and vascular regression in a wound remodeling phase. So, the mechanism of action of ozone may be associated with FGF2 overexpression and myofibroblastic differentiation (Soares et al., 2019).

Additionally, ozone activates the immune system by inducing secretion of interferon- $\gamma$ and tumor necrosis factor- $\alpha$, Interleukin 2 (IL-2) and other factors involved in the inflammatory response (Bocci 2006; 2007). The underlying mechanism involves the interaction of ozone with wound exudates, which leads to the breakdown of ozone into peroxides and stimulates tissue repair, improving oxygenation in the area (Xiao et al., 2017). Species of oxygen, released by the macrophages, stimulate platelet aggregation and lead to the release of growth factors, which aid in wound healing (Valacchi and Bocci, 1999). However, studies should be performed to highlight the phenotype of macrophages that produce and secrete FGF2 during wound healing (M1 or M2 macrophages). Characteristically, M1 
macrophages induce inflammation, negatively regulating the early healing process, producing IL-12 and M2 macrophages positively regulate the healing process in an IL-10 mediated process (Mills, 2012).

Ozone therapy has been shown to increase tissue oxygenation and metabolism, interfering with oxidant/antioxidant balance. Based on this mechanism, ozone is capable of reducing the levels of hydrogen peroxide $\left(\mathrm{H}_{2} \mathrm{O}_{2}\right)$ by regulating the levels of antioxidant molecules. Also, peroxides produced through ozone improve oxygen availability by regulating antioxidant molecules in red blood cells, favoring the metabolism and the release of cytokines, autacoids and growth factors that are fundamental in the treatment of metabolic, inflammatory and infectious diseases (Soares et al., 2019). However, this release of peroxides in a large amount can cause harm to humans, as is evidenced in some studies that show structural changes in lung tissue (Buell et al., 1965), low resistance to respiratory bacterial infection and reduced lung function (Holzman et al., 1968). In addition, a pre-clinical study on the reaction of ozone with amino acids indicates that cysteine and methionine are easily oxidized, as well as tryptophan, histidine, tyrosine, cystine, and phenylalanine (Mudd et al., 1969). This oxidation of amino acids can be responsible for the toxic reactions of ozone to plants and animals. The studies above were performed mainly at ozone levels between 1.0 and 5.0 ppm (Leh, 1973).

Despite the possible deleterious dose-dependent effect, ozone therapy can increase oxygen metabolism in erythrocytes, increasing the rate of glycolysis, consequently leading to increased oxygen release into the tissues (Di Filippo et al., 2015). Ozone increases oxidative pyruvate carboxylation and ATP production during the Krebs' cycle, reducing NADH and oxidizes cytochrome C. Indeed, it increases the production of enzymes such as glutathione peroxidase, catalase, and superoxide dismutase that serve as sequestrators of free radicals and protectors for healthy cells (Nathan, 2002).

Ozonization in asthmatic patients effectively reduces Immunoglobulin (Ig)E and HLA-DR levels, thus improving lung function and reducing related symptoms. A study suggested that the therapeutic efficacy of ozone in reducing $\mathrm{IgE}$ and inflammatory mediators are associated with its immunomodulating and regulating properties of the oxidative stress (Wang, 2018). Another study showed a decrease in IgE and HLA-DR levels in asthmatic patients treated with systemic ozone therapy with the consequent increase in lung function and improvement in the symptoms (Hernández Rosales et al., 2005).

Evidence shows that moderate oxidative stress induced by low doses of ozone activates nuclear factor erythroid 2-related factor 2 (Nrf2), suppresses transcriptional factor-kappa $\mathrm{B}(\mathrm{NF}-\kappa \mathrm{B})$ and reduces inflammatory responses. Over the past decade, evidence has suggested that Nrf2 activation induces transcription of Antioxidant Response Elements (ERA). ERA transcription leads to the production of antioxidant enzymes such as Superoxide Dismutase (SOD), Glutathione Peroxidase (GPx), Glutathione S-Transferase (GST), Catalase (CAT), Heme-Oxygenase-1 (HO-1), NADPH-Quinone Oxide reductase (NQO-1), phase 2 enzymes of drug metabolism and heat shock proteins. Thus, the Nrf2 system contributes to the protection against carcinogenesis, liver toxicity, chronic respiratory and inflammatory diseases, neuronal ischemia and renal problems. Systemic administration of ozone through ozonated saline could activate this mechanism ( $\operatorname{Re}$ et al., 2014). In a study by Pecorelli et al. (2013), it was shown that ozonated plasma can positively regulate HO-1 expression in endothelial cells and activate Nrf2 in dosedependent serum ozone concentration.

On the other hand, severe oxidative stress triggered by high concentrations of ozone activates NF- $\kappa$ B, leading to elevated inflammatory responses and tissue damage by the production of Cyclooxygenase 2 (COX2), Prostaglandin E2 (PGE2) and cytokines. So, the strength of oxidative stress determines the effectiveness and toxicity of ozone (Wang, 2018). This effect was shown on the skin, demonstrating that ozone is capable of interacting with skin antioxidant molecules (Thiele et al., 1997), besides causing an increase in the expression of the COX-2, with an increase in heat shock protein (HSP) (Valacchi et al., 2005). The ozone dose that can cause toxicity is not yet defined, varying according to the clinical condition, route of administration and the individual's own organism (Bocci, 2006).

Ozone inactivates bacteria, viruses, fungi, yeast, and parasites through different mechanisms. In bacteria, ozone disrupts the integrity of the bacterial cell wall through oxidized phospholipids and lipoproteins (Polydorou et al., 2012; Thanomsub et al., 2002). In fungi, ozone inhibits its growth, while in viruses, ozone damages the viral capsid, preventing contact between the virus and the cell. Cells vulnerable to virus invasion are coated with oxidation-susceptible enzymes and can be eliminated from the body by interacting with ozone (Gupta and Brintnell, 2013; Travagli et al., 2010).

\section{Main Challenge and Difficulties}

The biggest challenge is the lack of standardization of ozone doses used in the studies, many not even mentioning this concentration. This may be due to the fact that ozone is a very unstable gas, which makes it difficult to remain in vehicles and, consequently, to measure the dose (Bocci et al., 2011). Another problem is the fact that in many countries medicine does not consider the use of ozone as a therapy, which makes it difficult to develop clinical treatment protocols (Re et al., 2012). 


\section{Conclusion}

The use of ozone in clinical practice has numerous benefits in the treatment of various diseases, mainly through the modulation of the immune system and of the oxidant/antioxidant balance and is applied to treat a wide of clinical conditions, both on local skin and systemic. The significant routes of use include the topical, intrarectal, subcutaneous and intravenous routes, carried out by different vehicles, as the main ones being saline, water, and ozone oil. The main characteristic of ozonetherapy is the anticipation of the healing process, which is related to the increase of endogenous growth factors and in the increase of oxygenation of the affected area. As well as its physiologic effect, ozone also inactivates microorganisms by its oxidizing capacity. This review evidenced that the toxicity of this compound is inherent to the concentration of ozone, being a higher dose responsible for causing exacerbation of the immune system with detrimental effects and a lower dose contributing to the reduction of inflammatory responses and increasing the production of antioxidant enzymes, thus improving the treatment of a range of pathologies. Altogether, ozone as a therapy has been used successfully in a wide range of medical conditions, however, it should be used in the correct concentration, paying attention to the dose range to avoid possible adverse effects due to toxicity of high concentration.

\section{Acknowledgment}

The authors thank Coordenação de Aperfeiçoamento de Pessoal de Nível Superior (CAPES) - the Brazilian government funding agency, for the fellowships received.

\section{Funding Information}

FWB. and IVS received a master's degree and TSA received a postdoctoral fellowship from the Coordenação de Aperfeiçoamento de Pessoal de Nível Superior (CAPES) - the Brazilian government funding agency.

\section{Author's Contributions}

Conceptualization: APP and RAM; Literature review: APP, FWB, IVS, MAD and TSA; Validation: RAM; Writing - original draft: APP, FWB, IVS, MAD, and TSA; Review and editing: RAM and TSA.

\section{References}

Almaz, M.E. and I.S. Sönmez, 2015. Ozone therapy in the management and prevention of caries. J. Formosan Med. Assoc., 114: 3-11. DOI: $10.1016 /$ j.jfma.2013.06.020
Almeida, E., M. Regina, M. Aparecida, M.R. Assalin and M.A. Rosa et al., 2004. Wastewater treatment by oxidation with ozone. Quimica Nova, 27: 818-824. DOI: $10.1590 / \mathrm{S} 0100-40422004000500023$

Andrade, R.R., O.B. de Oliveira $\square$ Neto, L.T. Barbosa, I.O. Santos and C.F. de Sousa $\square$ Rodrigues et al., 2019. Effectiveness of ozone therapy compared to other therapies for low back pain: A systematic review with meta $\square$ analysis of randomized clinical trials. Brazilian J. Anesthesiol., 69: 493-501.

DOI: 10.1016/j.bjan.2019.06.007

Anzolin, A.P. and C.D. Bertol, 2018. Ozone therapy as an integrating therapeutic in osteoartrosis treatment: A systematic review. Brazilian J. Pain, 1: 171-175. DOI: 10.5935/2595-0118.20180033

Babaei-Ghazani, A., S. Najarzadeh, K. Mansoori, B. Forogh and S.P. Madani et al., 2018. The effects of ultrasound-guided corticosteroid injection compared to oxygen-ozone $\left(\mathrm{O}_{2}-\mathrm{O}_{3}\right)$ injection in patients with knee osteoarthritis: A randomized controlled trial. Clin. Rheumatol., 37: 2517-2527. DOI: $10.1007 / \mathrm{s} 10067-018-4147-6$

Biçer, Ş., I. Sayar, C. Gürsul, A. Işık and M. Aydın et al., 2016. Use of ozone to treat ileostomy dermatitis in an experimental rat model. Medical Sci. Monitor, 22: 757-765. DOI: 10.12659/MSM.897696

Bocci, V.A., 2006. Scientific and medical aspects of ozone therapy. State of the art. Arch. Med. Res., 37: 425-435. DOI: 10.1016/j.arcmed.2005.08.006

Bocci, V., 2007. The case for oxygen-ozonetherapy. Brit. J. Biomed. Sci., 64: 44-49. DOI: $10.1080 / 09674845.2007 .11732755$

Bocci, V., 2004. Ozone as Janus: This controversial gas can be either toxic or medically useful. Mediators Inflammat., 13: 3-11. DOI: $10.1080 / 0962935062000197083$

Bocci, V., E. Borrelli, I. Zanardi and V. Travagli, 2015. The usefulness of ozone treatment in spinal pain. Drug Design Dev. Therapy, 9: 2677-2685. DOI: $10.2147 /$ DDDT.S74518

Bocci, V., G. Venturi, M. Catucci, P.E. Valensin and M. Zazzi, 1998. Lack of efficacy of ozone therapy in HIV infection. Clin. Microbiol. Infect., 4: 667-669. DOI: $10.1111 / \mathrm{j} .1469-0691.1998 . t b 00355 . x$

Bocci, V., I. Zanardi, E. Borrelli and V. Travagli, 2012. Reliable and effective oxygen-ozone therapy at a crossroads with ozonated saline infusion and ozone rectal insufflation. J. Pharmacy Pharmacol., 64: 482-489. DOI: $10.1111 / j .2042-7158.2011 .01427 . x$

Bocci, V., I. Zanardi and V. Travagli, 2011. Oxygen/ozone as a medical gas mixture. A critical evaluation of the various methods clarifies positive and negative aspects. Med. Gas Res., 1: 6-6. DOI: 10.1186/2045-9912-1-6 
Bocci, V.A., 2006. Scientific and medical aspects of ozone therapy. State of the art. Arch. Med. Res., 37: 425-435.

DOI: $10.1016 /$ j.arcmed.2005.08.006

Borges, G.Á., S.T. Elias, S.M.M. da Silva, P.O. Magalhães and S.B. Macedo et al., 2017. In vitro evaluation of wound healing and antimicrobial potential of ozone therapy. J. CranioMaxillofacial Surgery, 45: 364-370.

DOI: $10.1016 / \mathrm{j} . j \mathrm{cms} .2017 .01 .005$

Borrelli, E., A. Diadori, A. Zalaffi and V. Bocci, 2012. Effects of major ozonated autohemotherapy in the treatment of dry age-related macular degeneration: A randomized controlled clinical study. Int. J. Ophthalmol., 5: 708-713.

DOI: $10.3980 /$ j.issn.2222-3959.2012.06.11

Braidy, N., M. Izadi, A. Sureda, N. Jonaidi-Jafari and A. Banki et al., 2018. Therapeutic relevance of ozone therapy in degenerative diseases: Focus on diabetes and spinal pain. J. Cellular Physiol., 233: 2705-2714. DOI: $10.1002 /$ jcp. 26044

Broadwater, W.T., R.C. Hoehn and P.H. King, 1973. Sensitivity of three selected bacterial species to ozone. Applied Microbiol., 26: 391-393.

Buell, G.C., Y. Tokiwa and P.K. Mueller, 1965. Potential crosslinking agents in lung tissue: Formation and isolation after in vivo exposure to ozone. Arch. Environ. Health: Int. J., 10: 213219. DOI 10.1080/00039896.1965.10663986

Calunga, J.L., S. Menéndez, R. León, S. Chang and D. Guanche et al., 2012. Application of ozone therapy in patients with knee osteoarthritis. Ozone: Sci. Eng., 34: 469-475.

DOI: $10.1080 / 01919512.2012 .719120$

Campanati, A., S. De Blasio, A. Giuliano, G. Ganzetti and K. Giuliodori et al., 2013. Topical ozonated oil versus hyaluronic gel for the treatment of partial- to full-thickness second-degree burns: A prospective, comparative, single-blind, non-randomised, controlled clinical trial. Burns, 39: 1178-1183.

DOI: 10.1016/j.burns.2013.03.002

Cardoso, C.R.B., A.M. Souza, E.A.V. Ferro, S. Favoreto and J.D.O. Pena, 2004. Influence of topical administration of n-3 and n- 6 essential and n-9 nonessential fatty acids on the healing of cutaneous wounds. Wound Repair Regenenerat., 12: 235-243. DOI: 10.1111/j.1067-1927.2004.012216.x

Celakil, T., A. Muric, B. Gokcen Roehlig, G. Evlioglu and H. Keskin, 2017. Effect of high-frequency bio-oxidative ozone therapy for masticatory muscle pain: a double-blind randomised clinical trial. J. Oral Rehabilit., 44: 442-451.

DOI: $10.1111 /$ joor. 12506
Clavo, B., D. Ceballos, D. Gutierrez, G. Rovira and G. Suarez et al., 2013. Long-term control of refractory hemorrhagic radiation proctitis with ozone therapy. J. Pain Symptom Manage., 46: 106-112. DOI: 10.1016/j.jpainsymman.2012.06.017

Coelho, C.S., J.A.N. Gama, L.A.T. Oliveira Júnior, B.S.F. Silva and V.R.C. Souza et al., 2012. Use of extracts of sunflower-seed oil (Helianthus annus L.) for the treatment of cutaneous injuries in equine metatarsus: A case report. Revista Brasileira Plantas Medicinais, 14: 125-129. DOI: 10.1590/S1516-05722012000100018

Coppola, L., B. Letieri, D. Cozzolino, C. Luongo and A. Sammartino et al., 2002. Ozonized autohaemotransfusion and fibrinolytic balance in peripheral arterial occlusive disease. Blood Coagulat. Fibrinolysis, 13: 671-681.

DOI: 10.1097/00001721-200212000-00002

Currò, M., T. Russo, N. Ferlazzo, D. Caccamo and P. Antonuccio et al., 2018. Anti-inflammatory and tissue regenerative effects of topical treatment with ozonated olive oil/vitamin E acetate in balanitis xerotica obliterans. Molecules, 23: 645-645. DOI: 10.3390/molecules23030645

da Silva, S.B., M.M. Luvielmo, M.C. Geyer and I. Pra, 2011. Potential use of ozone in the food processing. Semina: Ciências Agrárias, 32: 659-682. DOI: $10.5433 / 1679-0359.2011 v 32 n 2 p 659$

Delgado-Roche, L., M. Riera-Romo, F. Mesta, Y. Hernández-Matos and M. Barrios et al., 2017. Medical ozone promotes Nrf2 phosphorylation reducing oxidative stress and pro-inflammatory cytokines in multiple sclerosis patients. Eur. J. Pharmacol., 811: 148-154. DOI: 10.1016/j.ejphar.2017.06.017

Di Filippo, C., M. Luongo, R. Marfella, F. Ferraraccio and B. Lettieri et al., 2010. Oxygen/ozone protects the heart from acute myocardial infarction through local increase of eNOS activity and endothelial progenitor cells recruitment. NaunynSchmiedeberg's Arch. Pharmacol., 382: 287-291. DOI: $10.1007 / \mathrm{s} 00210-010-0545-2$

Di Filippo, C., M.C. Trotta, R. Maisto, D. Siniscalco and M. Luongo et al., 2015. Daily oxygen $/ \mathrm{O}_{3}$ treatment reduces muscular fatigue and improves cardiac performance in rats subjected to prolonged high intensity physical exercise. Oxidative Medicine Cellular Longevity, 2015: 1-11. DOI: $10.1155 / 2015 / 190640$

Di Paolo, N., V. Bocci, D.P. Salvo, F. Palasciano and M. Biagioli et al., 2005. Extracorporeal Blood Oxygenation and Ozonation (EBOO): A controlled trial in patients with peripheral artery disease. Int. J. Artificial Organs, 28: 1039-1050. DOI: $10.1177 / 039139880502801012$ 
Di Paolo, N., E. Gaggiotti and F. Galli, 2005. Extracorporeal blood oxygenation and ozonation: Clinical and biological implications of ozone therapy. Redox Report, 10: 121-130. DOI: $10.1179 / 135100005 X 38888$

El-Sawalhi, M.M., H.A. Darwish, M.N. Mausouf and A.A. Shaheen, 2013. Modulation of age-related changes in oxidative stress markers and energy status in the rat heart and hippocampus: A significant role for ozone therapy. Cell Biochem. Funct., 31: 518-525.

DOI: $10.1002 / \mathrm{cbf} .2930$

Elvis, A. and J. Ekta, 2011. Ozone therapy: A clinical review. J. Natural Sci. Biol. Med., 2: 66-66. DOI: $10.4103 / 0976-9668.82319$

Eriksson, M., R. Andersson and L. Kloo, 2007. Reaction of SDS with ozone and $\mathrm{OH}$ radicals in aqueous solution. Ozone: Sci. Eng., 29: 131-138. DOI: $10.1080 / 01919510601187053$

Eroglu, Z.T., B. Kurtis, H.A. Altug, S. Sahin and G. Tuter et al., 2018. Effect of topical ozonetherapy on gingival wound healing in pigs: Histological and immuno-histochemical analysis. J. Applied Oral Sci., 27: e20180015-e20180015.

DOI: $10.1590 / 1678-7757-2018-0015$

Fitzpatrick, E., O.J. Holland and J.J. Vanderlelie, 2018. Ozone therapy for the treatment of chronic wounds: A systematic review. Int. Wound J., 15: 633-644. DOI: $10.1111 /$ iwj.12907

Frosini, M., A. Contartese, I. Zanardi, V. Travagli and V. Bocci, 2012. Selective ozone concentrations may reduce the ischemic damage after a stroke. Free Radical Res., 46: 612-618. DOI: $10.3109 / 10715762.2012 .659247$

Garber, G.E., D.W. Cameron, N. Hawley-Foss, D. Greenway and M.E. Shannon, 1991. The use of ozone-treated blood in the therapy of HIV infection and immune disease. AIDS, 5: 981-984.

DOI: 10.1097/00002030-199108000-00009

Gautam, S., V. Rastogi, A. Jain and A.P. Singh, 2011. Comparative evaluation of oxygen-ozone therapy and combined use of oxygen-ozone therapy with percutaneous intradiscal radiofrequency thermocoagulation for the treatment of lumbar disc herniation. Pain Practice, 11: 160-166. DOI: $10.1111 / \mathrm{j} .1533-2500.2010 .00409 . \mathrm{x}$

Gulmen, S., T. Kurtoglu, I. Meteoglu, S. Kaya and H. Okutan, 2013. Ozone therapy as an adjunct to vancomycin enhances bacterial elimination in methicillin resistant Staphylococcus aureus mediastinitis. J. Surgical Res., 185: 64-69.

DOI: $10.1016 /$ j.jss.2013.05.085
Gupta, A.K. and W.C. Brintnell, 2013. Sanitization of contaminated footwear from onychomycosis patients using ozone gas: A novel adjunct therapy for treating onychomycosis and tinea pedis? J. Cutaneous Med. Surgery, 17: 243-249. DOI: $10.2310 / 7750.2012 .12068$

Gupta, G. and B. Mansi, 2012. Ozone therapy in periodontics. J. Med. Life, 5: 59-67.

Hassanien, M., S. Rashad, N. Mohamed, A. Elawamy and M.S. Ghaly, 2018. Non-invasive oxygen-ozone therapy in treating digital ulcers of patients with systemic sclerosis. Acta Reumatol. Portuguesa, 2018: 210-216.

Hernández Rosales, F.A., J.L. Calunga Fernández, J.T. Figueras, S.M. Cepero and A.M. Perdomo, 2005. Ozone therapy effects on biomarkers and lung function in asthma. Arch. Med. Res., 36: 549-554. DOI: 10.1016/j.arcmed.2005.04.021

Holzman, R.S., D.E. Gardner and D.L. Coffin, 1968. In vivo inactivation of lysozyme by ozone. J. Bacteriol., 96: 1562-1566.

Izadi, M., R. Kheirjou, R. Mohammadpour, M.H. Aliyoldashi and S.J. Moghadam et al., 2019. Efficacy of comprehensive ozone therapy in diabetic foot ulcer healing. Diabetes Metabolic Syndrome: Clin. Res. Rev., 13: 822-825.

DOI: $10.1016 /$ j.dsx.2018.11.060

Kim, J.G., A.E. Yousef and S. Dave, 1999. Application of ozone for enhancing the microbiological safety and quality of foods: A review. J. Food Protect., 62: 1071-1087. DOI: $10.4315 / 0362-028 X-62.9 .1071$

Le, G.A.O., 2012. The nursing experience of ozone therapy of psoriasis. Inner Mongolia Med. J., 11: 44-44.

Leh, F., 1973. Ozone. Properties, toxicity and applications. J. Chem. Educ., 50: 404-404.

DOI: $10.1021 /$ ed050p404

León Fernández, O.S., R. Viebahn-Haensler, G.L. Cabreja, I.S. Espinosa and Y.H. Matos et al., 2016. Medical ozone increases methotrexate clinical response and improves cellular redox balance in patients with rheumatoid arthritis. Eur. J. Pharmacol., 789: 313-318. DOI: 10.1016/j.ejphar.2016.07.031

Liu, J., P. Zhang, J. Tian, L. Li and J. Li et al., 2015. Ozone therapy for treating foot ulcers in people with diabetes. Cochrane Database Systematic Rev., 2015: CD008474-CD008474.

DOI: 10.1002/14651858.CD008474.pub2

Loegager, T., J. Holcman, K. Sehested and T. Pedersen, 1992. Oxidation of ferrous ions by ozone in acidic solutions. Inorganic Chem., 31: 3523-3529. DOI: 10.1021/ic00043a009 
Lopes de Jesus, C.C., F.C. Dos Santos, L.M.O.B. De Jesus, I. Monteiro and M.S.S.C. Sant'ana et al., 2017. Comparison between intra-articular ozone and placebo in the treatment of knee osteoarthritis: A randomized, double-blinded, placebo-controlled study. PLoS ONE, 12: 1-16. DOI: 10.1371/journal.pone.0179185

Martínez-Sánchez, G., S.M. Al-Dalain, S. Menéndez, L. Re and A. Giuliani et al., 2005. Therapeutic efficacy of ozone in patients with diabetic foot. Eur. J. Pharmacol., 523: 151-161. DOI: 10.1016/j.ejphar.2005.08.020

Martínez-Sánchez, G., L. Delgado-Roche, A. DíazBatista, G. Pérez-Davison and L. Re, 2012. Effects of ozone therapy on haemostatic and oxidative stress index in coronary artery disease. Eur. J. Pnarmacol., 691: 156-162.

DOI: 10.1016/j.ejphar.2012.07.010

Mauro, R.D., G. Cantarella, R. Bernardini, M. Di Rosa and I. Barbagallo et al., 2019. The biochemical and pharmacological properties of ozone: The smell of protection in acute and chronic diseases. Int. J. Molecular Sci., 20: 634-647. DOI: $10.3390 /$ ijms20030634

Menéndez, S., L. Falcón and Y. Maqueira, 2011. Therapeutic efficacy of topical OLEOZON ${ }$ in patients suffering from onychomycosis. Mycoses, 54: e272-e277.

DOI: $10.1111 / \mathrm{j} .1439-0507.2010 .01898 . x$

Mills, C., 2012. M1 and M2 macrophages: Oracles of health and disease. Critical Rev. Immunol., 32: 463-488.

DOI: $10.1615 /$ CritRevImmunol.v32.i6.10

Mirowsky, J.E., L.A. Dailey and R.B. Devlin, 2016. Differential expression of pro-inflammatory and oxidative stress mediators induced by nitrogen dioxide and ozone in primary human bronchial epithelial cells. Inhalation Toxicol., 28: 374-382. DOI: $10.1080 / 08958378.2016 .1185199$

Morris, J.C., 1971. Chlorination and disinfection - State fo the art. J. Am. Water Works Assoc., 63: 769-774. DOI: 10.1002/j.1551-8833.1971.tb02611.x

Mudd, J.B., R. Leavitt, A. Ongun and T.T. McManus, 1969. Reaction of ozone with amino acids and proteins. Atmos. Environ., 3: 669-681. DOI: 10.1016/0004-6981(69)90024-9

Nathan, C., 2002. Catalytic antibody bridges innate and adaptive immunity. Science, 298: 2143-2144. DOI: $10.1126 /$ science. 1080005

Niu, T., C. Lv, G. Yi, H. Tang and C. Gong et al., 2018. Therapeutic effect of medical ozone on lumbar disc herniation. Med. Sci. Monitor, 24: 1962-1969.

DOI: 10.12659/MSM.903243
Noori-Zadeh, A., S. Bakhtiyari, R. Khooz, K. Haghani and S. Darabi, 2019. Intra-articular ozone therapy efficiently attenuates pain in knee osteoarthritic subjects: A systematic review and meta-analysis. Complementary Therapies Med., 42: 240-247. DOI: 10.1016/j.ctim.2018.11.023

Nowicka, D., 2017. Thermography improves clinical assessment in patients with systemic sclerosis treated with ozone therapy. Biomed Res. Int., 2017: 1-7. DOI: $10.1155 / 2017 / 5842723$

Ohmuller, 1892. Action of ozone on bacteria. Arbeitskreis Gesundheit, 8: 228-235.

Oliveira, M.L.M., D.C.S. Nunes-Pinheiro, B.M.O. Bezerra, L.O. Leite and A.R. Tomé et al., 2013. Topical anti-inflammatory potential of pumpkin (Cucurbita pepo L.) seed oil on acute and chronic skin inflammation in mice. Acta Scientiae Vet., 41: 1168-1168.

Orakdogen, M., S. Uslu, S.T. Emon, H. Somay and Z.C. Meric et al., 2016. The effect of ozone therapy on experimental vasospasm in the rat femoral artery. Turkish Neurosurgery, 26: 860-865. DOI: 10.5137/1019-5149. JTN.14162-15.2

Ozturk, B., T. Kurtoglu, S. Durmaz, L.D. Kozaci and F. Abacigil et al., 2017. The effects of ozone on bacterial growth and thiol-disulphide homeostasis in vascular graft infection caused by MRSA in rats. Acta Cirurgica Brasileira, 32: 219-228. DOI: $10.1590 / \mathrm{S} 0102-865020170030000006$

Paoloni, M., L. Di Sante, A. Cacchio, D. Apuzzo and S. Marotta et al., 2009. Intramuscular oxygenozone therapy in the treatment of acute back pain with lumbar disc herniation: A multicenter, randomized, double-blind, clinical trial of active and simulated lumbar paravertebral injection. Spine, 3: 1337-1344.

DOI: $10.1097 /$ BRS.0b013e3181a3c18d

Pecorelli, A., V. Bocci, A. Acquaviva, G. Belmonte and C. Gardi et al., 2013. NRF2 activation is involved in ozonated human serum upregulation of HO-1 in endothelial cells. Toxicol. Applied Pharmacol., 267: 30-40. DOI: 10.1016/j.taap.2012.12.001

Polydorou, O., A. Halili, A. Wittmer, K. Pelz and P. Hahn, 2012. The antibacterial effect of gas ozone after 2 months of in vitro evaluation. Clin. Oral Invest., 16: 545-550. DOI: 10.1007/s00784-011-0524-0

Qin, G., J. Huang, Y. Pan, Y. Xiang and C. Ou et al., 2018. Topical ozone application: An innovative therapy for infantile atopic dermatitis. Zhong nan da xue xue bao. J. Central South Univ. Med. Sci., 43: $163-167$.

DOI: $10.11817 /$ j.issn. 1672-7347.2018.02.010 
Re, L., G. Malcangi and G. Martinez-Sanchez, 2012. Medical ozone is now ready for a scientific challenge: Current status and future perspectives. J. Exp. Integrative Med., 2: 193-196.

DOI: $10.5455 /$ jeim.070612.ir.012

Re, L., G. Martínez-Sánchez, M. Bordicchia, G. Malcangi and A. Pocognoli et al., 2014. Is ozone pre-conditioning effect linked to Nrf2/EpRE activation pathway in vivo? A preliminary result. Eur. J. Pharmacol., 742: 158-162.

DOI: 10.1016/j.ejphar.2014.08.029

Ripamonti, C.I., E. Cislaghi, L. Mariani and M. Maniezzo, 2011. Efficacy and safety of medical ozone $\left(\mathrm{O}_{3}\right)$ delivered in oil suspension applications for the treatment of osteonecrosis of the jaw in patients with bone metastases treated with bisphosphonates: Preliminary results of a phase I-II study. Oral Oncol., 47: 185-190.

DOI: 10.1016/j.oraloncology.2011.01.002

Rivas-Arancibia, S., R. Vazquez-Sandoval, D. GonzalezKladiano, S. Schneider-Rivas and A. LechugaGuerrero, 1998. Effects of ozone exposure in rats on memory and levels of brain and pulmonary superoxide dismutase. Environ. Res., 76: 33-39. DOI: 10.1006/enrs.1997.3784

Rodrigues, K.L., C.C. Cardoso, L.R. Caputo, J. Carlos and T. Carvalho et al., 2004. Cicatrizing and antimicrobial properties of an ozonised oil from sunflower seeds. InflammoPharmacology, 12: 261-270. DOI: 10.1163/1568560042342275

Sega, A., I. Zanardi, L. Chiasserini, A. Gabbrielli and V. Bocci et al., 2010. Properties of sesame oil by detailed ${ }^{1} \mathrm{H}$ and ${ }^{13} \mathrm{C}$ NMR assignments before and after ozonation and their correlation with iodine value, peroxide value and viscosity measurements. Chem. Phys. Lipids, 163: 148-156.

DOI: 10.1016/j.chemphyslip.2009.10.010

Sivalingam, V.P., E. Panneerselvam, K.V.B. Raja and G. Gopi, 2017. Does topical ozone therapy improve patient comfort after surgical removal of impacted mandibular third molar? A randomized controlled trial. J. Oral Maxillofacial Surgery, 75: 51.e1-51.e9. DOI: 10.1016/j.joms.2016.09.014

Smith, N., A. Wilson, J. Gandhi, S. Vatsia and S. Khan, 2017. Ozone therapy: An overview of pharmacodynamics, current research and clinical utility. Medical Gas Res., 7: 212-219.

DOI: $10.4103 / 2045-9912.215752$

Soares, C.D., T.M.L. Morais, R.M.F.G. Araújo, P.F. Meyer and E.A.F. Oliveira et al., 2019. Effects of subcutaneous injection of ozone during wound healing in rats. Growth Factors, 37: 95-103.

DOI: $10.1080 / 08977194.2019 .1643339$
Solovăstru, L.G., A. Stîncanu, A. De Ascentii, G. Capparé and P. Mattana et al., 2015. Randomized, controlled study of innovative spray formulation containing ozonated oil and $\alpha$-bisabolol in the topical treatment of chronic venous leg ulcers. Adv. Skin Wound Care, 28: 406-409.

DOI: 10.1097/01.ASW.0000470155.29821.ed

Stübinger, S., R. Sader and A. Filippi, 2006. The use of ozone in dentistry and maxillofacial surgery: A review. Quintessence Int., 37: 353-359.

Svystonyuk, D.A., J.M.C. Ngu, H.E.M. Mewhort, B.D. Lipon and G. Teng et al., 2015. Fibroblast growth factor-2 regulates human cardiac myofibroblast-mediated extracellular matrix remodeling. J. Translat. Med., 13: 147-147. DOI: $10.1186 / \mathrm{s} 12967-015-0510-4$

Sweet, F., M.S. Kao, S.C. Lee, W.L. Hagar and W.E. Sweet, 1980. Ozone selectively inhibits growth of human cancer cells. Science, 209: 931-933. DOI: 10.1126/science.7403859

Taşdemir, Z., B.A. Alkan and H. Albayrak, 2016. Effects of ozone therapy on the early healing period of deepithelialized gingival grafts: A randomized placebo-controlled clinical trial. J. Periodontol., 87: 663-671. DOI: 10.1902/jop.2016.150217

Thanomsub, B., V. Anupunpisit, S. Chanphetch, T. Watcharachaipong and R. Poonkhum et al., 2002. Effects of ozone treatment on cell growth and ultrastructural changes in bacteria. J. General Applied Microbiol., 48: 193-199. DOI: 10.2323/jgam.48.193

Thiele, J.J., M.G. Traber, K. Tsang, C.E. Cross and L. Packer, 1997. In vivo exposure to ozone depletes vitamins $\mathrm{C}$ and $\mathrm{E}$ and induces lipid peroxidation in epidermal layers of murine skin. Free Radical Biol. Med., 23: 385-391.

DOI: $10.1016 / \mathrm{S} 0891-5849(96) 00617-\mathrm{X}$

Travagli, V., I. Zanardi, G. Valacchi and V. Bocci, 2010. Ozone and ozonated oils in skin diseases: A review. Mediators Inflammation, 2010: 1-9.

DOI: $10.1155 / 2010 / 610418$

USFDA, 2019. Code of federal regulations title 21. U.S. Food and Drug Administration.

Valacchi, G. and V. Bocci, 1999. Studies on the biological effects of ozone: 10. Release of factors from ozonated human platelets. Mediators Inflammation, 8: 205-209.

DOI: $10.1080 / 09629359990360$

Valacchi, G., V. Fortino and V. Bocci, 2005. The dual action of ozone on the skin. Brit. J. Dermatol., 153: 1096-1100. DOI: 10.1111/j.1365-2133.2005.06939.x

Vanni, D., R. Galzio, A. Kazakova, A. Pantalone and A. Sparvieri et al., 2016. Intraforaminal ozone therapy and particular side effects: Preliminary results and early warning. Acta Neurochirurgica, 158: 491-496. DOI: 10.1007/s00701-015-2545-y 
Wainstein, J., Z. Feldbrin, M. Boaz and I. HarmanBoehm, 2011. Efficacy of ozone-oxygen therapy for the treatment of diabetic foot ulcers. Diabetes Technol. Therapeut., 13: 1255-1260.

DOI: $10.1089 /$ dia.2011.0018

Wang, X., 2018. Emerging roles of ozone in skin diseases. J. Central South Univ., 43: 114-123. DOI: $10.11817 / \mathrm{j}$. issn. 1672-7347.2018.02.002

Wysok, B., J. Uradziñski and M. Gomólka-Pawlicka, 2006. Ozone as an alternative disinfectant: A review. Polish J. Food Nutrit. Sci., 15: 3-8.

Xiao, W., H. Tang, M. Wu, Y. Liao and K. Li et al., 2017. Ozone oil promotes wound healing by increasing the migration of fibroblasts via PI3K/Akt/mTOR signaling pathway. Biosci. Rep., 37: 1-11. DOI: 10.1042/BSR20170658
Zaky, S., S.E. Kamel, M.S. Hassan, N.A. Sallam and M.A. Shahata et al., 2011. Preliminary results of ozone therapy as a possible treatment for patients with chronic hepatitis C. J. Alternative Complementary Med., 17: 259-263.

DOI: $10.1089 / \mathrm{acm} .2010 .0016$

Zeng, J. and J. Lu, 2018. Mechanisms of action involved in ozone-therapy in skin diseases. Int. Immunopharmacol., 56: 235-241. DOI: $10.1016 /$ j.intimp.2018.01.040

Zhang, J., M. Guan, C. Xie, X. Luo and Q. Zhang et al., 2014. Increased growth factors play a role in wound healing promoted by noninvasive oxygen-ozone therapy in diabetic patients with foot ulcers. Oxidative Med. Cellular Longevity, 2014: 273475-273475. DOI: $10.1155 / 2014 / 273475$ 